\title{
A Feminist Virtual Agent for Breastfeeding Promotion
}

\author{
Lin Shi ${ }^{1}$, Timothy Bickmore ${ }^{1}$, Roger Edwards ${ }^{2}$ \\ ${ }^{1}$ College of Computer and Information Science, Northeastern University, Boston, MA, USA \\ \{octlin, bickmore\}@ccs.neu.edu \\ ${ }^{2}$ College of Health Sciences, Northeastern University, Boston, MA, USA
}

\begin{abstract}
The design of a conversational virtual agent that plays the role of a lactation educator promoting breastfeeding is described, along with a manipulation to make the agent appear more or less feminist. Results from a randomized pilot study indicate that study participants were aware of the manipulation and participants who were more feminist preferred the feminist agent, while those who were non-feminist preferred the non-feminist agent. This work demonstrates one way that feminism can be incorporated into agent design, and a methodology for identifying users who will be most receptive to it.
\end{abstract}

Keywords: Feminism, embodied conversational agent, relational agent, breastfeeding.

\section{$1 \quad$ Introduction}

Culture as "the entire range of a society's arts, beliefs, institutions, and communication practices" [1] shapes gender --- one of the organizing principles of human society --- on a daily basis in both material as well as discursive ways. There is a significant amount of research on the cultural and social construction of gender roles [2-4]. Feminism is a collection of ideas and activities aimed at examining, revealing and overcoming "the economic, political, social, and psychological oppression of women" [1]. Academically speaking, feminists draw attention to the central of issues of gender and sexuality in the understanding of social experience and symbolic power in both the private as well as public sphere.

There has been a growing interest in incorporating feminist perspectives into technology design methodology --- one of the practices of "cultural" adaptation according to our understanding. A CHI 2011 workshop on "Feminist HCI" focused on aspects of value sensitive design. However, this "feminist $\mathrm{HCl}$ ", as admitted by the workshop participants, remains "a promising, yet underdeveloped term" [5]. Bardzell expounds that "the interaction design process takes place independent of gender considerations, and even today the central concept of the whole field - the user - remains genderless" [6].

In our work, we are interested in moving beyond only using feminist values in our design methodology, to incorporating these values into the technology artifacts themselves, so that users will recognize the artifacts and the messages they produce as feminist. In doing this, we are intentionally creating artifacts, such as virtual agents, 
that are "tailored" [7] for a very specific subset of the population. We are also investigating whether informational messages from the scientific frame, delivered by a virtual agent, are better received by women in general if they are re-framed from an inclusive feminist perspective.

To explore issues of feminism in technological artifacts in general, and virtual agents in particular, we have conducted our research in the domain of breastfeeding promotion. Breastfeeding in itself is an important health topic: many major US public health and medical organizations have been actively promoting breastfeeding and recommend exclusive breastfeeding for the first six months of life [8], and then "breastfeeding should be continued for at least the first year of life and beyond for as long as mutually desired by mother and child"[8]. Despite these proclamations by the medical establishment, only $16.3 \%$ of mothers in the US follow this recommendation, leading to a range of interventions to improve breastfeeding rates [9]. However, breastfeeding represents a controversial issue from the academic feminist perspective. The cultural consensus that "breast is best" is based on the scientific evidence of breastfeeding's biological and psychological benefits to both babies and their mothers, while an alternative perspective sees this medical "prescription" as subjugating mothers" "rights" to their children's perceived "needs." There is an emerging voice shared by feminist theorists [10-12], who are concerned more about women's freedom and autonomy, that breast-feeding advocacy should move beyond the debate over the medical effects of breastfeeding, and instead focus on securing social resources that enable women's freedom to make and enact their decisions about infant feeding.

In the rest of this paper we describe the design and implementation of a virtual agent that plays the role of a lactation educator whose job is to motivate new mothers to breastfeed exclusively for six months (Figure 1). We describe the development of two versions of this agent: one that frames its arguments exclusively from the scientific/medical perspective, and one that provides the same argumentation but frames it from the feminist perspective. We then describe preliminary results from a study in which we compared women's reactions to the two versions of the agent.

\section{Design of a Feminist Virtual Agent for Breastfeeding Promotion}

The initial version of the virtual agent was developed to play the role of a virtual lactation educator. Our development methodology involved initially videotaping sample counseling sessions with a certified lactation consultant. This was followed by several months of meetings of an interdisciplinary design team to work through the overall design of the system and the specific dialogue scripts and media content used in each part of the intervention. We first designed a 20-minute interaction intended to motivate women in their third trimester to follow the CDC recommendations. The topics covered in this script include: greeting; asking user about her most important breastfeeding topic; review of the CDC recommendations; review of benefits of breastfeed- 
ing for the baby; breastfeeding benefits for the mother; breastfeeding "101" (latching, basic nursing positions); review of CDC recommendations; and farewell.

The virtual agent's appearance was designed based on feedback from user testing with new mothers (Figure 1). The agent is rendered using the LiteBody framework [13], with speech output produced with a commercial speech synthesizer. Dialogues are scripted using a custom hierarchical transition network-based scripting language. Agent nonverbal conversational behavior is generated using BEAT [14], and includes beat (baton) hand gestures and eyebrow raises for emphasis, a range of iconic, emblematic, and deictic gestures, gaze away behavior for signaling turn-taking, and posture shifts to mark topic boundaries, synchronized with speech. User input is obtained via multiple choice selection of utterances [15].

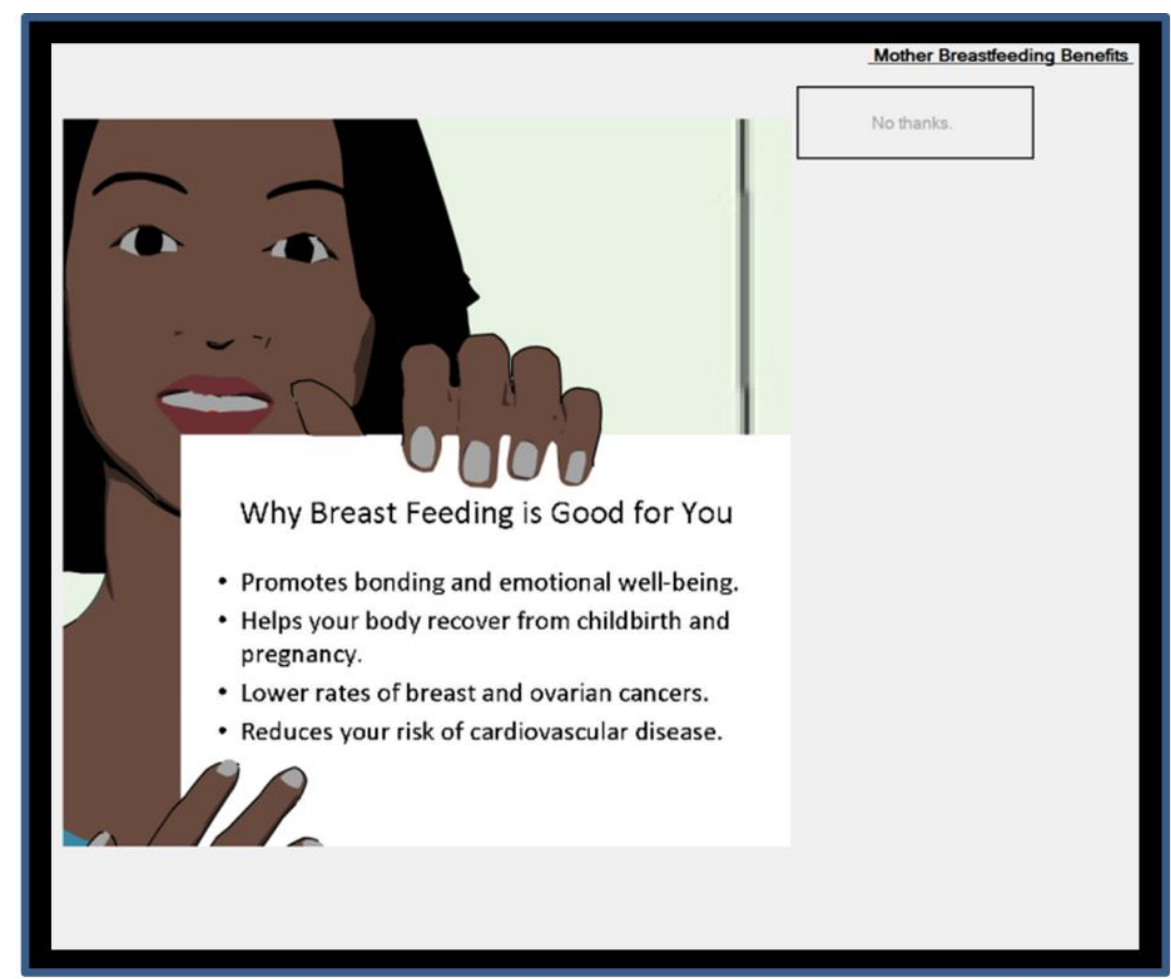

Fig. 1. Virtual Agent Interface Used in Breastfeeding Promotion System

We next sought to create a version of the agent that delivered the same information content, but in a way that would be recognized as feminist by most women. We began by reviewing relevant literature [16-19], videotapes of lectures given by experts on the topic of feminism and breastfeeding, and tape-recorded and transcribed interviews with a convenience sample of self-identified feminists who were breastfeeding advocates. We included some elements of "second wave" feminism (anti-patriarchy), but focused the messaging on themes of "third wave" feminism (equality and inclusion) $[20,21]$. An initial introductory script and closing script were drafted to precede and 
follow the medical agent script (just after the greeting and before the closing, relative to Figure 2, respectively) without modifying the medical content delivered. This initial script was sent to several women for review and revised based on their feedback. The final version of the feminist scripts are shown in Figure 2.

\section{Feminist Introduction Script}

1. A: I like to think of myself as a fairly progressive woman, and I hope I do not offend you with some of my opinions. I will just be as honest as I can, with you. I totally respect any choice that anybody makes. I am not going to tell any woman what she should or should not do with her body. I can tell you what I think, but whatever you choose is your choice. But, I think you should make that choice in an informed way. There actually is a lot of science behind the benefits to you and your baby, that we will talk about today...

\section{Feminist Closing Script}

1. A: You know, for me, in addition to all that important scientific stuff, there's one thing that really tipped my opinion in favor of breastfeeding. Once I realized that breastfeeding is something that is not done by most women in our society, and that huge formula corporations are actively working against it, it really brought out the rebel in me. I like doing nontraditional things, especially when it comes to women's issues. I would like to think that if women were not being allowed into universities, I would be one of those ones knocking down the doors. I like doing things that are not traditional for women. That's part of my identity, it's who I am. ...

2. A: Breastfeeding is a learned thing. It's not something that you are born knowing how to do. For thousands of years, women have learned this from other women. Unfortunately, we don't have the community of women that we used to have. That's another thing that's been taking away from us, thank you very much capitalist patriarchy. It's something that you should learn from a circle of aunts, and girlfriends, and grandmothers.

3. A: It really makes me angry when hospitals send new mothers home with infant formula and advertisements, because it short-circuits that learning process that has to happen. It makes it so that you cannot make that informed choice, that I think is important.

4. A: A lot of people think that breastfeeding represents a dilemma for feminism.

5. A: You know, should women minimize gender differences as the path to liberation, or should they embrace gender differences by fighting to remove the constraints placed on them by patriarchy and capitalism? I can't say what feminism is about, because there are as many kinds of feminism as there are feminists. But for me, the reason that many feminist philosophies appeal to me, is that it's about choice and freedom. It's about choice that is truly informed, and about removing barriers to that choice...

Fig. 2. Feminist Script Excerpts

A: Agent utterance; U: User utterance

\section{Feminist Agent Acceptance Study}

In order to compare women's reactions to the feminist vs. medical breastfeeding promotion agent, we conducted a randomized between-subjects study in which partici- 
pants were randomized into three groups: MEDICAL, in which the agent presented the scientific/medical arguments for breastfeeding; FEMINIST, in which the agent presented both the medical arguments and the feminist introduction and closing (Figure 2); and CONTROL, in which women did not receive any intervention. In this paper we are focusing only on results regarding attitudes towards the FEMINIST agent compared to the MEDICAL agent. Each of these conversations took approximately 20 minutes to complete, after which participants filled out a questionnaire about their attitudes towards the agent and conducted a semi-structured interview about their experience. Our hypotheses follow tailoring theory [7], in that we expect participants with stronger feminist orientations to prefer the FEMINIST agent, whereas those with weaker feminist orientations will prefer the MEDICAL agent.

\subsection{Measures}

In addition to sociodemographics, we measured feminist self-identification [22] and feminist values [23] at intake, using validated 7-point Likert scale instruments. Following interaction with the agent, we assessed overall satisfaction, desire to continue, comfort discussing breastfeeding with the agent, and preference to discuss breastfeeding with a doctor or nurse (rather than the agent), in addition to assessing the degree to which the agent was feminist (as a manipulation check), Table 1.

\begin{tabular}{|l|c|l|c|c|c|}
\hline \multicolumn{1}{|c|}{ Question } & Anchor 1 & \multicolumn{1}{|c|}{$\begin{array}{c}\text { Anchor } \\
\mathbf{7}\end{array}$} & $\begin{array}{c}\text { MEDICAL } \\
\text { mean (sd) }\end{array}$ & $\begin{array}{c}\text { FEMINIST } \\
\text { mean (sd) }\end{array}$ & p \\
\hline $\begin{array}{l}\text { How satisfied were you with } \\
\text { Tanya? }\end{array}$ & not at all & $\begin{array}{l}\text { very } \\
\text { satisfied }\end{array}$ & $5.81(1.08)$ & $4.87(1.21)$ & $\mathbf{. 0 0 4}$ \\
\hline $\begin{array}{l}\text { How much would you like to } \\
\text { continue working with } \\
\text { Tanya? }\end{array}$ & not at all & $\begin{array}{l}\text { very } \\
\text { much }\end{array}$ & $5.07(1.27)$ & $4.19(1.57)$ & $\mathbf{. 0 2 6}$ \\
\hline $\begin{array}{l}\text { How comfortable were you } \\
\text { discussing breastfeeding with } \\
\text { Tanya? }\end{array}$ & not at all & $\begin{array}{l}\text { very } \\
\text { much }\end{array}$ & $5.68(1.81)$ & $5.93(1.11)$ & .545 \\
\hline $\begin{array}{l}\text { Would you have rather heard } \\
\text { this information from a doc- } \\
\text { tor or nurse? }\end{array}$ & not at all & $\begin{array}{l}\text { very } \\
\text { much }\end{array}$ & $3.96(1.53)$ & $4.00(1.49)$ & .929 \\
\hline $\begin{array}{l}\text { How would you characterize } \\
\text { Tanya as a feminist? }\end{array}$ & $\begin{array}{c}\text { very anti- } \\
\text { feminist }\end{array}$ & $\begin{array}{l}\text { very } \\
\text { feminist }\end{array}$ & $4.3(1.03)$ & $5.93(1.07)$ & $<.001$ \\
\hline
\end{tabular}

Table 1. Self-Report Questions and Responses ("Tanya" is the name of agent)

\subsection{Results}

Nulliparous females (women who have not had any children) were recruited through an online job posting service (craigslist), fliers posted around the university, and a local newspaper. Nulliparous women were selected, since we felt attitudes towards breastfeeding of those who already had children would be relatively more so- 
lidified and so would be less receptive to new information. Seventy-two (72) women completed the study. Participants were 23.9 (sd 4.1) years old, $63 \%$ white, $21 \%$ black, $89 \%$ never married, $46 \%$ were students and $90 \%$ had at least some collegelevel education. Overall, participants scored on the lower ends of the feminist identity (mean 3.2, sd 1.1) and feminist values (mean 3.3, sd 0.5) scales. Feminist identity and values were highly correlated, Pearson $\mathrm{r}=.67, \mathrm{p}<.001$. We created bivariate factors for feminist identity and feminist values by splitting women into two groups for each measure at the median.

Table 1 shows the ratings of the MEDICAL and FEMINIST agents by all participants. Participants did rate the FEMINIST agent as being more feminist than the MEDICAL agent, $\mathrm{t}(52)=5.7, \mathrm{p}<.001$, establishing that our manipulation was effective. Overall, participants were significantly less satisfied with the FEMINIST agent, $\mathrm{t}(52)=3.0, \mathrm{p}<.01$, and were less inclined to continue working with it, $\mathrm{t}(52)=2.3, \mathrm{p}<.05$, compared to the MEDICAL agent.

We also performed 2x2 non-parametric ANOVAs (using the Aligned Rank Transform [8]) with agent type (MEDICAL vs. FEMINIST) and participant feminist orientation (feminist identity or feminist values category) as factors.

While women overall were less satisfied with the FEMINIST agent, those women who did not self-identify as feminist were less satisfied with the FEMINIST agent, $\mathrm{F}(1,50)=3.1, \mathrm{p}=.08$ (trending), and were significantly less likely to continue working with it, $\mathrm{F}(1,50)=4.3, \mathrm{p}<.05$, compared to the MEDICAL agent, while women who self-identified as feminist were much less dis-satisfied with the FEMINIST agent (Figure 3).

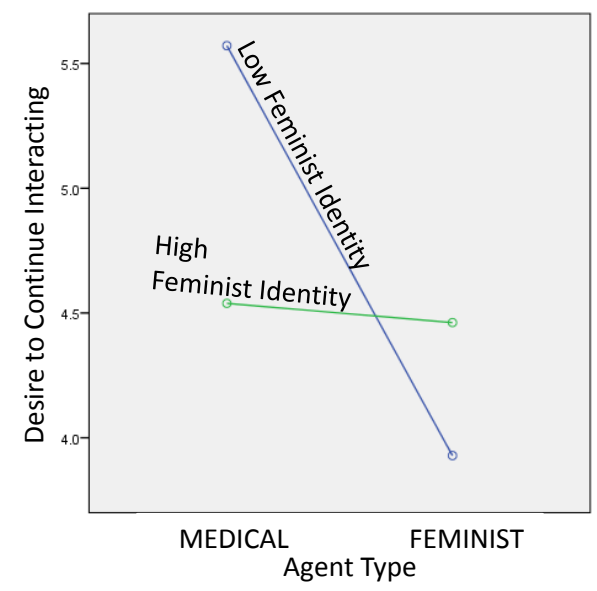

Figure 3. Interaction of Agent Type and Feminist Identity on Desire to Continue

There was also a significant interaction between agent type and feminist values, such that women who endorsed feminist values were more comfortable talking with the FEMINIST agent about breastfeeding, while women who did not endorse feminist values were more comfortable talking with the MEDICAL agent, $F(1,51)=3.6, p=.06$. Similarly, women who endorsed feminist values preferred talking to the FEMINIST 
agent rather than a doctor or nurse about breastfeeding, while women who did not endorse feminist values preferred receiving this information from the MEDICAL agent, $F(1,50)=3.8, \mathrm{p}=.06$ (Figure 4 ).
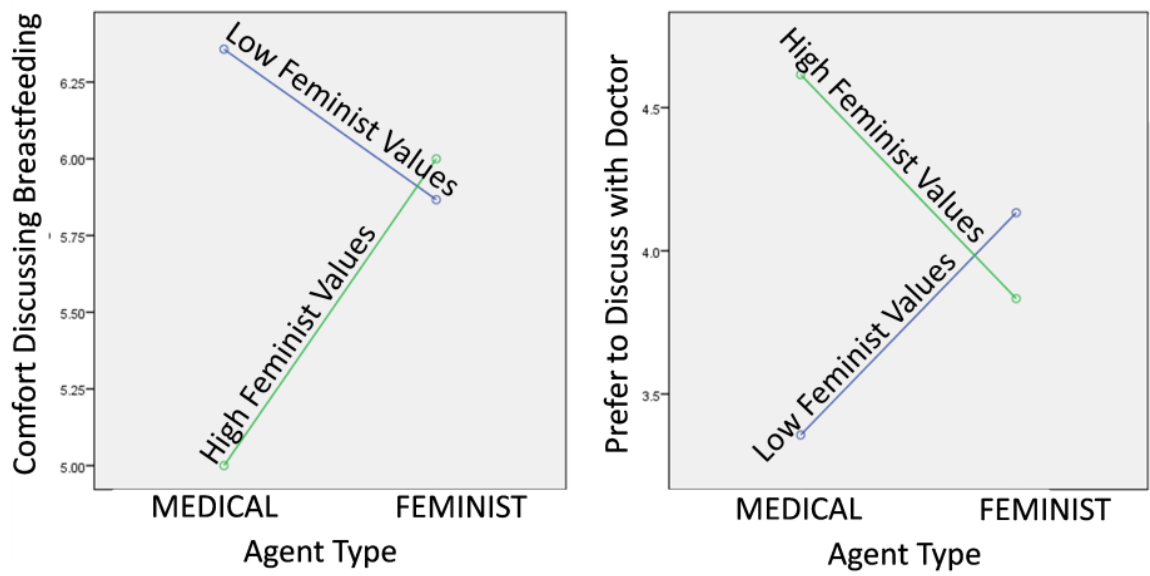

Fig. 4. Interaction of Agent Type and Feminist Values on Comfort Discussing Breastfeeding with Agent (L) and Preference for Talking to Doctor or Nurse about Breastfeeding (R)

\subsection{Interview Results from Participants in FEMINIST Condition}

Most comments about the agent's feminist language were from low-feminist participants, and were mostly negative.

Participant 022:

Q: "What about other things you might have disliked about Tanya?"

A: "Uhh, well she's so feminist, just at the end, all this stuff, it was too much for me. But that's ok."

\section{Participant 051:}

Q: "Ok. Did anything about it seem strange to you?"

A: "I thought, just like Tanya, how she was very feminist, like that kind of turned me off."

Most high-feminist participants did not notice or comment on the agent's feminist language. The few who did said they felt it did not affect them.

\section{Participant 046:}

Q: "Did you find her persuasive?"

A: "Umm, actually, the speech at the end, I could pick up on a little bit of feminism going on there, umm, but it didn't seem to affect me in any way. Because I focused on the information ... but you know, it was like, she didn't say anything that offended me, so I didn't take offense in any way, so."

One high-feminist participant touched on feminist themes in noting that it was good to get breastfeeding information from "another woman". 


\section{Participant 042:}

Q: "Ok. What did you think about Tanya specifically?"

A: "Umm, I think she, as like a computer character, was kinda, very relatable. Umm, I was kind of expecting to see like a man, as the system. So it was nice as a woman hearing from another woman about how to breastfeed because it seems like these things are always dominated by, or are always a male role."

\subsection{Discussion}

Study participants who interacted with the FEMINIST agent rated it as being more feminist compared to participants who interacted with the MEDICAL agent, thus our manipulation worked. We also observed anticipated interaction effects between participant feminist orientation and the type of agent on their attitudes towards the agent, with feminist participants preferring the FEMINIST agent and non-feminist participants preferring the MEDICAL agent. However, we also observed lower satisfaction scores for the FEMINIST agent across all participants.

One reason for the overall lower satisfaction with the FEMINIST agent may simply have to do with the increased interaction time due to the additional feminist scripts. Also, since this part of the interaction was largely a monologue and had little information content, some participants may have felt that this part of the interaction was a waste of their time. It is also possible that the young women in our study follow the "depoliticized and individualistic" norm of young women in the US who agree with feminist ideas but do not label them (or themselves) as such [24].

This study had many limitations, beyond the very small and likely biased sample of study participants. There are many interpretations of feminism and we certainly only captured a few of them in our agent's feminist dialogue scripts. Achieving the feminist manipulation by simply "bookending" the medical dialogue script is an oversimplistic approach: a much better approach would be to use a dialogue system that is capable of interleaving feminist messages throughout the medical dialogue, or a text generator that is able to infuse all medical messages with feminist values, rather than abruptly switching frames. Finally, the FEMINIST condition was significantly longer than the MEDICAL condition, and the increased session duration, exposure to the agent, and messaging about breastfeeding all represent potential confounds for our study design.

\section{Conclusion}

We designed a virtual agent that plays the role of a lactation educator promoting breastfeeding, and a manipulation to make the agent appear more or less feminist in its language. Results from the pilot study indicate that participants were aware of the manipulation, and those who were more feminist preferred the feminist agent, while those who were non-feminist preferred the non-feminist agent. 


\subsection{Future Work}

A multidisciplinary team has expanded the MEDICAL version of the virtual lactation educator into a full-blown, six-month duration intervention to promote breastfeeding, designed to be used in the obstetrician's office in the third trimester, in the labor and delivery ward of the hospital, and at home six months after birth. A pilot study on its efficacy has been conducted [25] and researchers are currently running a six-month randomized clinical trial with 84 women to evaluate its long-term impact [26].

There are significant opportunities to further explore feminist virtual agents, given the plethora of literature and theorizing on the topic, as well as further exploration of feminism as a persuasive message tailoring variable. More generally, the automatic generation (or recognition) of messages that frame scientific "facts" from different political points of view (as in [27]) is a potentially important and intriguing direction for future research, an initial step toward a stronger modeling-based approach.

\section{References}

1. Nelson, C., Treichler, P.A., Grossberg, L.: Cultural studies: An introduction. Cultural studies 1, 22 (1992)

2. Lorber, J.: The social construction of gender. The social construction of difference and inequality: Race, class, gender, and sexuality 99-106 (2003)

3. Davis, T.L.: Voices of gender role conflict: The social construction of college men's identity. Journal of College Student Development (2002)

4. Lorber, J.: Paradoxes of gender. Yale University Press (1994)

5. Bardzell, S., Churchill, E., Bardzell, J., Forlizzi, J., Grinter, R., Tatar, D.: Feminism and interaction design. In: Conference Feminism and interaction design, pp. 1-4. ACM, (2011)

6. Bardzell, S.: Feminist HCI: taking stock and outlining an agenda for design. In: Conference Feminist HCI: taking stock and outlining an agenda for design, pp. 1301-1310. ACM, (2010)

7. Hawkins, R.P., Kreuter, M., Resnicow, K., Fishbein, M., Dijkstra, A.: Understanding tailoring in communicating about health. Health education research 23, 454-466 (2008)

8. American Academy of Pediatrics: (2005)

9. Centers for Disease Control and Prevention: Breastfeeding report card - United States, 2014. (2014)

10. Hausman, B.L.: Mother's milk: Breastfeeding controversies in American culture. Psychology Press (2003)

11. Hausman, B.L.: Breastfeeding, Rhetoric, and the Politics of Feminism. Journal of Women, Politics \& Policy 34, 330-344 (2013)

12. Wolf, J.B.: Is breast best?: taking on the breastfeeding experts and the new high stakes of motherhood. NYU Press (2010)

13. Bickmore, T., Schulman, D., Shaw, G.: DTask \& LiteBody: Open Source, Standards-based Tools for Building Web-deployed Embodied Conversational Agents. Intelligent Virtual Agents, Amsterdam, Netherlands (2009) 
14. Cassell, J., Vilhjálmsson, H., Bickmore, T.: BEAT: The Behavior Expression Animation Toolkit. In: Conference BEAT: The Behavior Expression Animation Toolkit, pp. 477-486. (2001)

15. Bickmore, T., Picard, R.: Establishing and Maintaining Long-Term HumanComputer Relationships. ACM Transactions on Computer Human Interaction 12, 293-327 (2005)

16. Blum, L.M.: At the breast: Ideologies of breastfeeding and motherhood in the contemporary United States. Beacon Press (2000)

17. Baumslag, N., Michels, D.L.: Milk, money, and madness: The culture and politics of breastfeeding. Bergin \& Garvey Westport (1995)

18. Drouin, K.H.: The situated mother: Evolutionary theory and feminism as complementary components to understanding breastfeeding behavior. Journal of Social, Evolutionary, and Cultural Psychology 7, 326 (2013)

19. Blum, L.M.: Mothers, babies, and breastfeeding in late capitalist America: The shifting contexts of feminist. (1993)

20. Bardzell, S.: Feminist HCI: Taking Stock and Outlining an Agenda for Design. ACM Conference of Human Factors in Computing Systems (CHI) (2010)

21. Krolokke, C., Sorensen, A.S.: Gender communication theories and analyses: From silence to performance. Sage (2006)

22. Szymanski, D.: Relations among dimensions of feminism and internalized heterosexism in lesbians and bisexual women. Sex Roles 51, 145-159 (2004)

23. Fischer, A., Tokar, D., Mergl, M., Good, G., Hill, M., Blum, S.: Assessing women's feminist identity development: Studies of convergent, discriminant, and structural validity. Psychology of Women Quarterly 24, 15-29 (2000)

24. Aronson, P.: Feminists Or "Postfeminists"? Young Women's Attitudes toward Feminism and Gender Relations. Gender \& Society 17, 903-922 (2003)

25. Edwards, R.A., Bickmore, T., Jenkins, L., Foley, M., Manjourides, J.: Use of an Interactive Computer Agent to Support Breastfeeding. Maternal and child health journal 17, 1961-1968 (2013)

26. Zhang, Z., Bickmore, T., Mainello, K., Mueller, M., Foley, M., Jenkins, L., Edwards, R.A.: Maintaining Continuity in Longitudinal, Multi-method Health Interventions Using Virtual Agents: The Case of Breastfeeding Promotion. In: Conference Maintaining Continuity in Longitudinal, Multi-method Health Interventions Using Virtual Agents: The Case of Breastfeeding Promotion, pp. 504-513. Springer, (2014)

27. Hovy, E.: Generating Natural Language Under Pragmatic Constraints. Lawrence Erlbaum Associates (1988) 